www.jmscr.igmpublication.org

Index Copernicus Value: 79.54

ISSN (e)-2347-176x ISSN (p) 2455-0450

crossrefDOI: https://dx.doi.org/10.18535/jmscr/v7i3.45

\title{
Prevalence of cheiroarthropathy in Type2 Diabetes Mellitus in a Tertiary care hospital
}

\author{
Authors \\ Dr Jacob Antony ${ }^{1}$, Dr Ratheesh Narayanan Santhanavally ${ }^{2 *}$ \\ ${ }^{1,2}$ Associate Professor, Department of Internal Medicine, Government Medical College, Thiruvanathapuram, \\ Kerala, India \\ *Corresponding Author \\ Dr Ratheesh Narayanan Santhanavally \\ Email: ratishkarthika@yahoo.co.in
}

\section{Introduction}

India has nearly 66.8 million cases of diabetes, and these numbers are expected to rise to 120.9 million in 2035. India is set to become the diabetic capital of the world. Once thought of as an urban disease, now a day's diabetes has become highly prevalent in rural population also. The prevalence of diabetes is significantly high in south Indian population. The reasons for increasing prevalence are not exactly known. However, physical inactivity, dietary indiscretion and genetic factors may be playing a role in the rising prevalence of diabetes in India. The pathogenesis of diabetes is also highly complex. There are many defects: impaired insulin secretion, insulin resistance, in cretin defects, hypererglucogonemia and increased reabsorbption of glucose from kidneys. Because of these multiple defects in diabetes, it is very difficult to manage a diabetic patient and achieve targets of glycemic control. To compound this, diabetics will usually have associated comorbidities like dyslipidemia, obesity and hypertension also. Diabetes predisposes to microvascular complications (nephropathy, retinopathy and neuropathy) and macrovascular complications (coronary artery disease, cerebrovascular disease and peripheral vascular disease. Indian diabetics are more prone for complications ${ }^{2}$. The exact reasons behind the increased prevalence of diabetic complications are not very clear. Genetic factors may be playing a role.

Diabetes predisposes to other complications also. They include infections, gastrointestinal complications and musculoskeletal complications. Though data regarding the typical micro and macrovascular complications are available in abundance, data regarding these atypical manifestations are not that widely available. These complications may not be producing mortality like coronary artery disease or cerebrovascular disease, but they produce significant morbidities and makes the patient's life miserable. These complications are often under recognised and underreported. Musculoskeletal manifestations of diabetes include adhesive capsulitis of shoulder, flexor tenosynovitis, shoulder hand syndrome, chiroarthropthy, Dupuytrenes contracture etc. 
These manifestations produce significant morbidity for the patient. We always tend to ignore the impact of these complications on the personal and social life of a diabetic patient. The etiopathogenesis of musculoskeletal complications is similar to other complications. It is chiefly the AGEs and polyol pathway playing the role in the causation of these complications. Hyperglycemia may accelerate non-enzymatic glycosylation and abnormal collagen deposition in periarticular connective tissues, which alters the structural matrix and mechanical properties of these tissues leading to diffuse arthrofibrosis. So naturally it can be assumed that these complications are related to the duration of diabetes and degree of glycemic control. Proper history and physical examination often leads to the diagnosis of these conditions. Some simple lab tests and radiological examinations will help us in confirming the diagnosis and excluding any other co-existing rheumatologic disease. Once we diagnose these conditions, these patients can be helped by achieving and maintaining euglycemic state, analgesics when required and physiotherapy. As we know, the treatment options are limited. So the best treatment strategy would be preventive- to prevent the occurrence of these complications by aggressively controlling hyperglycemia and other co-morbidities from the beginning. Since there are not much data regarding this aspect of diabetes from this part of the country, we thought we will conduct a study on this particular complicationmusculoskeletal complications- at an era in which diabetes is growing in epidemic proportions. The chief aim of the study was to find out the prevalence of these chiroarthropathy in the OPD setting of a tertiary referral hospital and to find out the association between these complications and the duration and degree of glycemic control.

\section{Cheiroarthropathy (Limited Joint Mobility)}

Since the initial description of striking limitation of extension and flexion of the IP, MP, and wrist and other large joints associated with, thick tight waxy skin and early microvascular complications in three older teenagers with longdiabetes ${ }^{3}$.limited joint mobility (LJM) has been recognized as a common early complication of both type1 DM and type-2 $\mathrm{DM}^{4,5}$. Milder manifestations than those originally reported have been described in one fourth to one half of patients with type $1 \mathrm{DM}$ and in one of fourth to three fourth of subjects with type $2 \mathrm{DM}$. Depending on the age of the population the duration of the diabetes, and the examination techniques. In older patients, however, observations are confounded by the fact that as many as $20 \%$ of the adult non diabetic population may behave finger contracture related to age, occupation, or non-diabetes-associated problems, such as osteoarthritis. There are studies showing association between retinopathy and hand changes in type $1 \mathrm{DM}^{6}$

\section{Clinical Characteristics}

Changes begin in the MP and proximal IP joints of the fifth finger and Extend radially with involvement of the distal IP joints as well. Larger joints maybe involved, most commonly the wrist and elbow but also the ankles and the cervical and thoracolumbar spine. The limitation is painless and only mildly disabling when severe. There is no associated muscle atrophy ${ }^{4,7}$. Involvement Of the cervical spine can complicate endotracheal intubation for anaesthesia ${ }^{8}$, and foot involvement can contribute to abnormal foot pressure anddamage ${ }^{9}$. diminished pulmonary capacity also has been noted, but it remains uncertain whether this is caused by decreased pulmonary compliance or thoracic joint limitation ${ }^{10}$. Thick tight waxy skin most prominent over the dorsum of the Hands and forearms was noted in the original patients and in one third of those Subsequently described, but only in the presence of moderate or severe joint changes. Skin biopsy specimens show active fibroblasts and extensive collagen polymerization in the rough endoplasmic reticulum. There is a predominance of large fibers. This was also found in patients with diabetes whether or not they had thick skin, which is dissimilar from the bimodality of collagen fiber sizes in scleroderma. limited joint mobility in children and adolescents seems to be associated 
with growth delay, even in milder forms. The patients originally described were severely growth impaired. In a large cross-sectional study, 38\% of patients without LJM had statures below the twenty-fifth percentile (versus an expected 25\%), whereas $75 \%$ to $80 \%$ of those with mild and moderate/severe limitation were below the twenty-fifth percentile for height. There is also evidence for association with microvascular complications.

\section{Examination and classification}

The extent of joint involvement is easily evaluated by the "prayer sign" or the "table test". With the prayer sign, the patient is asked to touch the palmar surfaces of the interphalangeal joints together with the fingers fanned. If he or she is unable to do so, the test is considered positive for cheiroarthropathy. This can be confirmed by passive extension of the fingers. The table test is positive if

The palmar surface of the hand, with fanned fingers, does not touch when pressed against a flat surface. A slightly more complicated version of the table test involves painting the patient's palmar surfaces. The patient then pushes both palms firmly down onto a sheet of paper on a flat surface to make an imprint. This quickly identifies any areas that do not make contact. The tissue surrounding the limited joints is often thickened, and there is an inability to tent the skin over the dorsum of the fingers and hand as well as the elbow to at least 180 degrees; the ankles should flex maximally to at least 100degrees; and cervical spine lateral flexion should permit ear-toshoulder juxtaposition and thoracolumbar spine lateral flexion to at least 35 degrees in young persons ${ }^{16}$ Joint mobility can be classified according to test results as follows:

Stage 0: ability to make contact at all opposing interphalangeal joints in the prayer sign or on the imprint

Stage 1: inability to make contact at one intetphalangeal joint in the prayer

Sign or on the imprint
Stage 2: inability to make contact at two or more interphalangeal joint in the prayer sign or on the imprint

\section{Natural History}

Attained age seems to be more important than duration of diabetes or age of onset of diabetes in the evolution of $\mathrm{LJM}^{13}$. In children, the interval between the detection of mild LJM and the progression to moderate or severe changes ranges from 3 months to 4 years with a mean of 2 years, following which progression, if any, is slow. Mild changes often persist. Two thirds of adult patients have at least two fingers involved ${ }^{16}$. Whereas half of young patients with more than 5 years duration of diabetes have moderate or severe limitation ${ }^{13}$. Association of Limited Joint Mobility with Long Term Complications of Diabetes The initial report of the prevalence of LJM included seven patients with severe changes of whom five and clinically apparent retinopathy before the age of 18 years ${ }^{4}$. In a longitudinal study of 169 patients with diabetes duration greater than 4.5 years (the shortest duration at which complications were noted), LJM was found to be associated with an $83 \%$ risk for retinopathy or nephropathy after 16 years of diabetes, whereas the risk was $25 \%$ in the absence ofLJM ${ }^{17}$.Several subsequent studies have described similar increased risk ${ }^{18-19}$.

Significant correlation is also found between LJM and microvascular disease in Type $2 \mathrm{DM}$, but the association is less significant than with type1 $\mathrm{DM}^{20}$. Prolonged nerve conduction velocity for the ulnar and median nerves in older patients with LJM has been noted compared with those without LJM and comparable duration of diabetes ${ }^{18}$. In a large series from the Joslin Clinic, LJM was found to be associated with a 4.3-folder relative risk of clinical neuropathy in type $1 \mathrm{DM}^{19}$. An 83 per cent risk for microvascular complication after 16 years of diabetes if joint limitation was present, but only a 25 per cent risk if joint limitation was absent. Consequently, limited joint mobility identifies a population exceptionally at risk for the early development of microvascular complications. 


\section{Aetiology of Cheiroarthropathy}

The etiology of cheiroarthropathy in diabetes is not well understood, but changes in the affected joints appear to be similar to those that occur with other chronic complications of the disease. Impaired joint mobility could be due to changes in the structure of the hand (eg, intrinsic muscle, joint capsule, subcutaneous tissue) or to alterations in extrinsic structures (eg: long flexor muscles, flexor tendons of the forearm). There are several explanations for these changes. First, changes may be explained by vascular ischemia caused by microangiopathy of dermal and subcutaneous vessels, which can cause fibrosis of connective tissue. The joint contractures are similar to those seen in early systemic sclerosis, which are secondary to generalized vascular changes and appear as waxy and tight skin on the fingers. Collagen abnormalities also may be involved in the etiology. This is suggested by evidence of (1) bowed fingers and reduced finger extension, which can be corrected by full flexion of the wrist, as seen in patients with collagen infiltration of forearm flexor muscles in systemic sclerosis (2) increased non-enzymatic glycosylation of collagen secondary to hyperglycemia, which can lead to lesions of the joints , capsules, and skin; and (3) increased crosslinkage of collagen, which has been observed in experimental diabetes and can be reversed by penicillamine therapy. Abnormalities in aldose reductase pathways are another possible cause of cheiroarthropathy. In diabetic patients, the increased formation of sorbitol and other polyols due to saturation of the sorbitol dehydrogenase pathway results in movement of water into cells and intracellular swelling. This, in turn, leads to skin thickening, periarticular stiffness, and reduced joint mobility, Finally neuropathic changes may be involved,. Evidence suggests that joint contractures in diabetic in diabetic patients are linked with delayed median nerve conduction and intrinsic hand-muscle wasting.

\section{Prevalence}

Cheiroarthropathy occurs in $4 \%$ to $14 \%$ of the non diabetic population.

In the diabetic population, the reported incidence varies widely: $8.4 \%$ to $55 \%$ with type 1 diabetes and $40 \%$ to $76 \%$ with type 2 diabetes. Several explanations have been suggested to account for these extreme variations. First, the differences may be related to patient selection. Second, diagnosis of limited joint mobility may be based on subjective criteria. Third, genetic or racial factor may be explain some differences, such as the $4.2 \%$ incidence reported in Italy ${ }^{4}$ versus the $16 \%$ incidence in Nigerian patient ${ }^{1}$ with type 2 diabetes. Finally, the duration of diabetes varies among study groups, and the prevalence of cheiroarthropathy increases with the duration of diabetes. In 2 Indian studies, the prevalence of 22 and $16 \%$ respectively. In a study from UK, Prevalence of LJM was found to be lower than 20 years ago and that improved standards of glycemic control and diabetes care may have contributed to this occurence.

\section{Management}

There is no well-established treatment for managing cheiroarthropathy, but physical therapy is important to maintain range of motion and prevent further deterioration. Since increased nonenzymatic glucosylation of collagen secondary to hyperglycemia Is considered to be one of the main cause of diabetic cheiroarthropathy. It makes sense to maintain the best glycemic control possible. A number of complement therapies have been tried in patients with diabetic cheiroarthropathy. However, most are anecdotal or experimental.

Injection of long-acting corticosteroid into the affected flexor tendon sheaths has been found to be effective ${ }^{7}$. Surgical intervention with tenolysis also has led to improvement in affected joints .Treatment with aldose reductase inhibitors has been investigated89. Use of sorbinil resulted in dramatic improvement in three diabetic cheiroarthropathy patients (35 to 63 years of age), each of whom had hand stiffness and severe grip 
weakness for more than a year. Improvement was evident within 4 weeks of initiation of therapy, and no complications were observed. However, sorbinil hasnot been marketed because of hepatotoxicity. Ponalrestat was used in a doubleblind, placebo-controlled in 13 patients 18 to 25 years of age who had diabetic cheiroarthropathy. It failed to improve joint mobility after 6 months 90. Pencillamine and betaaminopropionitrile can reverse increased collagen cross-linkage seen in experimental diabetes in animals ${ }^{11}$, but these drugs have not been used in diabetic patients with cheiroarthropathy. None of the drugs have been proved tobe absolutely useful in diabetic cheiroarthropathy. But the most effective method to prevent this troubling manifestation will be good glycemic control.

\section{Objectives}

1. To assess the prevalence of cheiroarhropathy in type 2 diabetes among the patient attending in diabetic clinic of Govt Medical College Thiruvanathapuram.

2. To find out the association between chiroarthropathy and the duration of diabetes mellitus.

3. To find out the association between chiroathroapthy and the degree of glycemic control.

\section{Methodology}

\section{Materials \& Methods}

Study Design: Cross sectional, observational study

Study Setting: Diabetic clinic of Government medical college Thiruvanathapuram.

Study Subjects: 150 consecutive diabetic patients attending diabetic clinic

Inclusion criteria: Type 2 Diabetic patients above 30years of age.

\section{Exclusion criteria}

1. Patients with known rheumatological diseases

2. Patients with significant renal / hepatic impairment
3. Acutely ill patients

4. Pregnancy

Study Period: 1 year (2015-2016)

Sample Size and Sampling: Sample size was calculated using the formula $(\mathrm{Z} \alpha)^{2} \mathrm{PQ} / \mathrm{d}^{2} \mathrm{P}=43.3$ $\%$ ( prevalence of joint manifestation in diabetic patient as per a study done in kerala) $60 \mathrm{Q}=100$ $\mathrm{P}=56.7 \mathrm{~d}$ =allowable error of $20 \% \mathrm{~d}=20 \%$ of $\mathrm{P}=8.6$

Sample $\quad$ size $=[3.84 \times 43.3 \times 56.7] / \quad 8.6 \times 8.6=132$ Minimum 132 consecutive cases of diabetic patient attending in diabetic clinic are needed for the study to be valid. So 150 consecutive cases of diabetic patient attending in diabetic clinic was examined

\section{Methodology}

After taking written informed consent, data was collected using a semi-structured questionnaire by interview technique. It contained detailed history regarding duration and degree of glycemic control. Specific questions were asked about joint symptoms and recorded. Symptoms of neuropathy were specifically asked and recorded.. In addition to general examination, anthropometry (including BMI) and vital statistics, detailed rheumatology examination was conducted - chiefly inspection and palpation of joints. The joint mobility of hands was assessed by prayer sign. Detailed neurological examination was done to exclude associated neuropathy. FBS, PPBS and HBAIC were be done to assess the present and past glycemic control, S. Cr and LFT were done to exclude co- morbid illness, CBC,ESR, RA factor and Uric acid were done to exclude any preexisting rheumatological disease in patients who had rheumatological manifestations. $X$ rays of hand, shoulder, spine and other involved joints were done to assess bone and joint changes in affected patients.

Variables under Study- musculoskeletal manifestation of diabetic patients, duration of diabetes mellitus, present glycemic control,

Ethical Concerns- Study started only after getting approval of institutional research 


\section{JMSCR Vol||07||Issue||03||Page 249-257||March}

committee and ethical committee. Informed consent was taken from each participant.

Statistical Analysis: Data were entered into Microsoft Excel and analysis done using SPSS software. Quantities variables are expressed in terms of Mean, Standard deviation and qualitative variables will be in analyzed using proportions. Relation between the variables were analyzed using Chi square. For test of significance $\mathrm{p}$ value $<0.05$ was considered statistically significant.

\section{Results}

A Cross-sectional study was done in Diabetic clinic of Government Medical College
Thiruvanathapuram among 150 consecutive diabetic patients.

Socio Demographic Profile of the study Subjects

Table 1: Age wise distribution of study participants

\begin{tabular}{|l|c|c|}
\hline Age & group & Number $\%$ \\
\hline $30-34$ & 6 & 4 \\
\hline $35-44$ & 22 & 14.7 \\
\hline $55-64$ & 28 & 18.7 \\
\hline $55-64$ & 59 & 39.3 \\
\hline$>64$ & 35 & 23.3 \\
\hline total & 150 & 100 \\
\hline
\end{tabular}

Figure 1: Age wise distribution of study participants

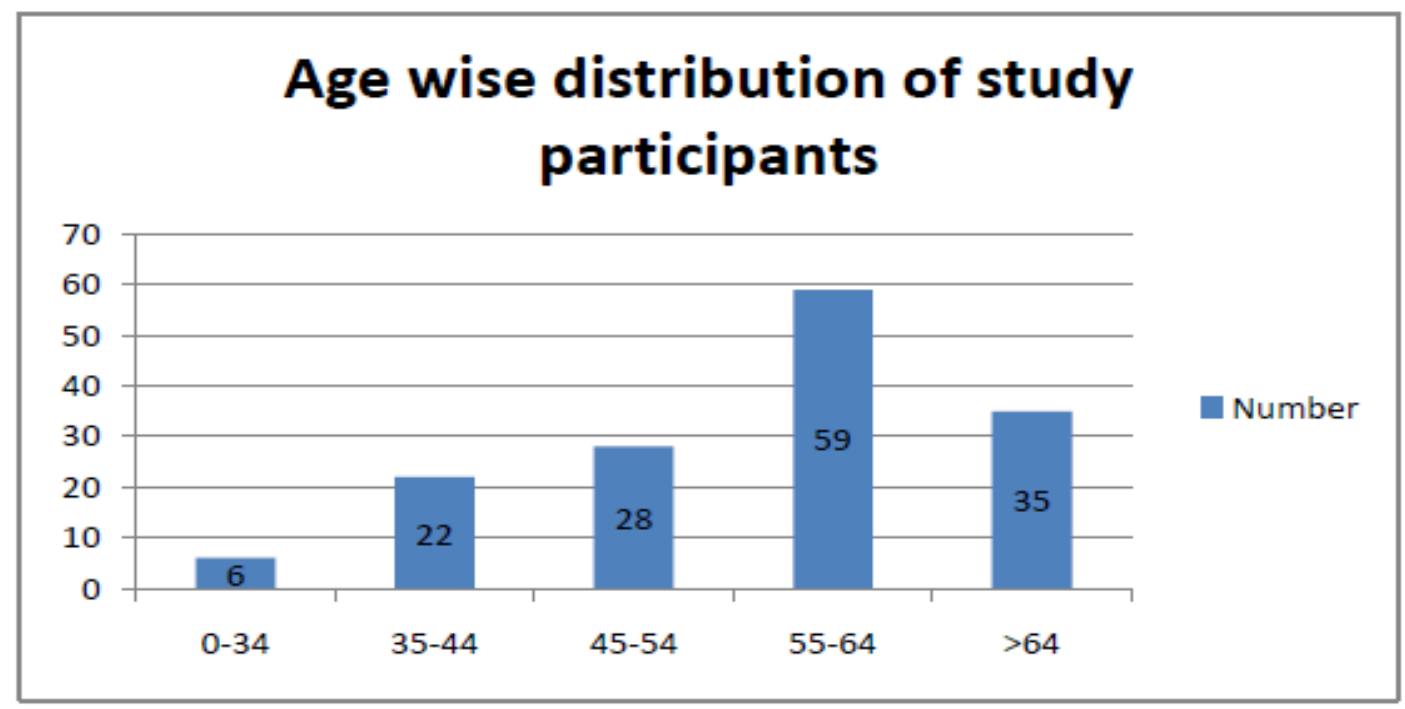

Figure 1 Age wise distribution of study participants

Mean age (SD) of study participants were 56 (11.4). It was seen that $39.3 \%$ of study subjects fall in the age group 55-64 age. There is also a tendency for the earlier occurrenec of diabetes ( $28 \%$ less than 45 years of age).

Table 2: Gender wise distribution of study subjects

\begin{tabular}{|l|c|c|}
\hline SEX & Number & $\%$ \\
\hline Male & 47 & 31.3 \\
\hline female & 103 & 68.7 \\
\hline total & 150 & 100 \\
\hline
\end{tabular}


Figure 2: Gender wise distribution of study subjects

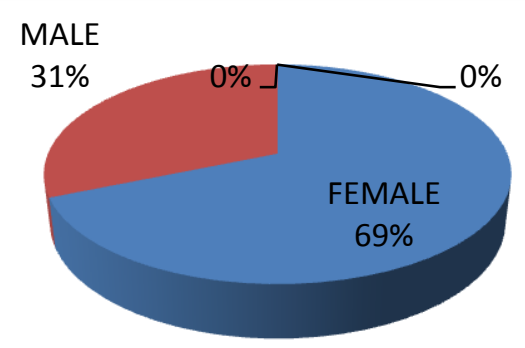

\section{Majority are females}

Table 3: Distribution of Study participants based Cheiroarthropathy manifestation

\begin{tabular}{|l|r|r|}
\hline \multicolumn{3}{|c|}{ Cheiropathy } \\
\hline Present & 8 & 5.3 \\
\hline Absent & 142 & 94.7 \\
\hline
\end{tabular}

Cheiroarthropathy was seen in $>5 \%$ of patients.

Table 4: HbA1c and Cheiroarthropathy

\begin{tabular}{|c|c|c|c|c|c|c|}
\hline \multicolumn{7}{|c|}{ Cheriopathy } \\
\hline HBA1CGP & Present(\%) & Absent(\%) & Total(\%) & $\begin{array}{l}\text { chi-square } \\
\text { value }\end{array}$ & D F & $\mathrm{P}$ value \\
\hline $\mathrm{Up}$ to 7 & 0 & $45(31.7)$ & $45(30)$ & & & \\
\hline $7.1 \mathrm{~T} 08$ & $1(12.5)$ & $36(25.4)$ & $37(24.7)$ & & & \\
\hline 8.1 TO 10 & $7(87.5)$ & $54(38)$ & $61(40.7)$ & & & \\
\hline $\begin{array}{l}\text { More than } \\
10\end{array}$ & 0 & $7(4.7)$ & $7(4.9)$ & & & \\
\hline Total & $8(100)$ & $142(100)$ & $150(100)$ & 7.99 & 3 & 0.04 \\
\hline
\end{tabular}

This depicts the relationship between $\mathrm{HbA}_{1 \mathrm{C}}$ and Cheiroarthropathy among the 8patients with Cheiroarthropathy. Among eight persons with
Cheiroarthropathy seven were having a $\mathrm{HbA} 1 \mathrm{C}$ of 8.1 to 10 and this association was found to be statically significant $(\mathrm{p}=0.04)$.

Table 5: Association between present FBS level and chiroarthropathy

\begin{tabular}{|c|c|c|c|c|c|c|}
\hline \multicolumn{7}{|c|}{ Cheriopathy } \\
\hline FBS & Present & Absent & Total & $\begin{array}{l}\text { chi-square } \\
\text { value }\end{array}$ & D F & $P$ value \\
\hline upto $130 \mathrm{mg}$.dl & $1(12.5)$ & $50(35.2)$ & 51934) & & & \\
\hline$>130 \mathrm{mg} / \mathrm{dl}$ & $7(87.5)$ & $92(64.8)$ & $99(66)$ & 1.74 & 1 & 0.87 \\
\hline Total & $8(100)$ & $142(100)$ & $150(100)$ & & & \\
\hline
\end{tabular}

Current FBS level has found out that not much significance with various musculoskeletal manifestations. 
Table 6: Association between PPBS level and Cheiroarthropathy

\begin{tabular}{|c|c|c|c|c|c|c|}
\hline \multicolumn{7}{|c|}{ Cheriopathy } \\
\hline PPBS & Present & Absent & Total & $\begin{array}{l}\text { chi-square } \\
\text { value }\end{array}$ & D F & $P$ value \\
\hline$<180 \mathrm{mg} / \mathrm{dl}$ & 0 & $30(21.1)$ & $30(20)$ & & & \\
\hline $180-240 \mathrm{mg} / \mathrm{dl}$ & $4(50)$ & $44(31)$ & $48(32)$ & 2.55 & 2 & 0.279 \\
\hline$>240 \mathrm{mg} / \mathrm{dl}$ & $4(50)$ & $68 \$ 7.9)$ & $72(48)$ & & & \\
\hline Total & $8(100)$ & $142(100)$ & $150(100)$ & & & \\
\hline
\end{tabular}

It has been obvious that long term glycemic control have more association than short term association.

Table 7: Association between duration of DM and Cheiroarthropathy

\begin{tabular}{|c|c|c|c|c|c|c|}
\hline \multicolumn{7}{|c|}{ Cheiropathy } \\
\hline Duration of DM & Present(\%) & Absent(\%) & Total (\%) & $\begin{array}{l}\text { chi- } \\
\text { square } \\
\text { value }\end{array}$ & D F & $P$ value \\
\hline Up to 5 year & 0 & $55(38.7)$ & $55(36.7)$ & & & \\
\hline 6 to 10 year & $2(25)$ & $42(29.6)$ & $44(29.3)$ & & & \\
\hline 11 to 15 year & $1(12.5)$ & $22(15.5)$ & $23(15.3)$ & & & \\
\hline $15-20$ year & $3(37.5)$ & $12(8.5)$ & $15(10)$ & & & \\
\hline $\begin{array}{c}\text { More than } 20 \\
\text { year }\end{array}$ & $2(25)$ & $11(7.7)$ & $13(8.7)$ & & & \\
\hline Total & $8(100)$ & $142(1000$ & $150(100)$ & 12.81 & 4 & 0.016 \\
\hline
\end{tabular}

It has been found that Cheiroarthropathy has a statistically significant association with duration of DM $(\mathrm{p}=0.016)$.Among the 8 persons withCheiroarthropathy3 were in the category of 15 to 20 years. Nobody developed Cheiroarthropathy within 5 years of onset.

\section{Discussion}

We conducted the study to find out the prevalence of chiroarthropathy and its relation to duration and degree of glycemic control. It was conducted in the setting of diabetic clinic of medical college. The very reason why we opted for this study is the high prevalence of diabetes in our community and lack of sufficient data regarding this particular diabetic complication. There are sufficient data regarding other complications like nephropathy and retinopathy. But regarding this disabling complications, no much data is available.

Majority of the study participants were females. Regarding $\mathrm{HBA}_{\mathrm{IC}}$ targets, more than $50 \%$ had HBA $_{\text {IC }}$ more than 8 .We have seen that a large number of patients are having musculoskeletal manifestations (44\%). Among the other manifestations, diabetic cheiroarthropathy as manifested by positive prayer sign was seen in $5.3 \%$ of the patients. There was statistically significant correlation between $\mathrm{HBA}_{\mathrm{IC}}$ and musculoskeletal manifestations -Cheiropathy. Then we tried to analyse the relation between current glycemic parameters and musculoskeletal manifestations. Both fasting glucose and post prandial glucose had a significant influence on the prevalence of musculoskeletal manifestations, re- 
establishing the fact that glycemic control current and past- is extremely important in preventing the onset of musculoskeletal manifestations. When we analyse the musculoskeletal manifestations, it was seen that 14 out of 15 diabetics who had diabetes more than 20 years duration had musculoskeletal manifestations, reemphasizing the fact that these manifestations are related to duration of diabetes.

\section{Conclusions}

Prevalence of musculoskeletal manifestations among diabetics is very high in South Kerala. [>40] Among the other manifestations, diabetic cheiroarthropathy as manifested by positive prayer sign was seen in $5.3 \%$ of the patients. There was statistically significant correlation between $\mathrm{HBA}_{\mathrm{IC}}$ and cheiroarthropathy.

There is a strong direct association between duration of diabetes and musculoskeletal manifestations.

\section{Bibliography}

1. International Diabetes Federation: IDF Diabetes Atlas Sixth Edition. 2013. Update 2014. $\quad$ www.idf.org/sites/default/files/ Atlas- poster-2014_EN.pdf (accessed December 22, 2015).

2. India Diabetes Management Algorithm proposal group, A proposed India specific algorithm for management of type 2 Diabetes, Diabetes technology and therapeautics, 1-5,Volume 18, Number 5, 2016

3. Rosenbloom AL, Frias JL: et al: Diabetes, short stature and joint stiffness - a new syndrome. Clin Res 22:92A,1974.

4. Grgic A, Rosenbloom AL, Weber FT, et al: Joint contracture-common manifestation of childhood diabetes mellitus. J Pediatr 8:584,1976.
5. Kennedy L, Beacom R, Archer DB, et al: Limited joint mobility in type I diabetes mellitus. Postgrad Med J 58:481,1982.

6. Lawson PM, Maneschi F, Kohner EM: The relationship of hand abnormalities todiabetes and retinopathy ,Diabetes Care 6:140,1983.

7. Rosenbloom AL: Limited joint mobility in insulin dependent childhood diabetes.Eur $\mathbf{J}$ Pediat 149:380,1992.

8. Salzarulo H, Taylor LA: Diabetic — stiff hand syndrome - as a cause of difficult intubation. Anaesthesia 64:366,1986.

9. Fernando DJS, Masson EA, Veves A, et al: Relationship of limited joint mobilityto abnormal foot pressure and diabetic foot ulceration. Diabetes Care 14:8,1991.

10. Schnapf BM, Banks R, Silverstein JH, et al: Pulmonary function in insulin dependent diabetes mellitus with limited joint mobility. Am Rev Respir Dis 130:930,1984. 